\title{
Prevalência de parasitoses intestinais e condições de saneamento básico das moradias em escolares da zona urbana de Caxias do Sul, Rio Grande do Sul
}

\author{
Prevalence of intestinal parasites among schoolchildren and household sanitation \\ in the urban area of Caxias do Sul, State of Rio Grande do Sul, Brazil
}

Jéssica Tadiello Camello', Natália Inês Cavagnolli², Patrícia Kelly Wilmsen Dalla Santa Spada ${ }^{3}$, Julia Poeta ${ }^{4}$, Adriana Dalpicolli Rodrigues ${ }^{5} \bowtie$

\author{
1 Laboratório de Análises Clínicas do Hospital Virvi Ramos. Caxias do Sul, RS. \\ 2 Universidade de Caxias do Sul. Caxias do Sul, RS. \\ ${ }^{3}$ Curso de Biomedicina, Enfermagem e Nutrição da Faculdade da Serra Gaúcha (FSG). Caxias do Sul, RS. \\ ${ }^{4}$ Curso de Biomedicina da UniRitter. Porto Alegre, RS. \\ ${ }^{5}$ Laboratório Alfa Ltda. Caxias do Sul, RS.
}

\section{RESUMO}

Objetivos: Avaliar a prevalência de parasitas intestinais em escolares de Caxias do Sul, relacionando-a com as condições de saneamento das moradias e com o conhecimento sobre parasitoses autorrelatado pelos familiares.

Métodos: Foram incluídas no estudo crianças com idade entre cinco e 13 anos, de escolas municipais de ensino fundamental localizadas em zona urbana de Caxias do Sul, Rio Grande do Sul, incluindo a periferia e as zonas centrais da cidade. As amostras foram processadas por sedimentação espontânea e analisadas microscopicamente. Os responsáveis pelos escolares responderam a um questionário elaborado para identificar condições socioeconômicas da família e de saneamento ambiental, assim como a impressão que os mesmos tinham sobre os seus conhecimentos acerca de parasitoses.

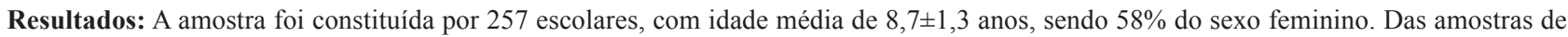
fezes analisadas, 5,8\% foram positivas para parasitas, sendo 60\% cistos de Endolimax nana, 26,7\% de Entamoeba coli e 13,3\% de Giardia lamblia. Com respeito às condições de vida dos escolares, $99,2 \%$ consumiam água potável, $94,6 \%$ afirmaram ter coleta e tratamento de esgoto e $99,2 \%$ contava com coleta pública de lixo na moradia. Sobre o conhecimento de parasitoses, $74,7 \%$ dos responsáveis sabiam o que são parasitas e $67,3 \%$ relataram que conheciam os meios de transmissão. No entanto, 49,8\% consideravam as informações insuficientes para a prevenção de parasitoses.

Conclusões: $O$ estudo evidenciou uma baixa prevalência de parasitoses nos escolares e condições favoráveis de saneamento em suas moradias. Houve relato de grande frequência de uso de medicamentos antiparasitários sem diagnóstico prévio por exame de fezes.

DESCRITORES: parasitas; parasitoses intestinais; crianças; escolares; saneamento básico; saneamento urbano.

\section{ABSTRACT}

Aims: To evaluate the prevalence of intestinal parasites among schoolchildren from Caxias do Sul, State of Rio Grande do Sul, southern Brazil, associating it with household sanitation and with family members' self-reported knowledge about parasites.

Methods: Children aged 5 to 13 years attending local elementary schools in the urban area of Caxias do Sul, including the outskirts and downtown area, were included in the study. The samples were processed by spontaneous sedimentation and analyzed microscopically. The children's legal representatives answered a questionnaire on socioeconomic conditions and sanitation as well as on their knowledge about parasitic infections.

Results: The sample included 257 schoolchildren with a mean age of $8.7 \pm 1.3$ years, most of whom were female (58\%). Of the analyzed stool samples, $5.8 \%$ were positive for parasitic cysts: Endolimax nana (60\%), Entamoeba coli (26.7\%), and Giardia lamblia (13.3\%). Regarding living conditions, $99.2 \%$ of the schoolchildren had access to treated water, $94.6 \%$ said they had sewage collection and treatment in their households, and $99.2 \%$ reported having garbage collection. As to legal representatives' knowledge about parasitic infections, $74.7 \%$ knew what they were and $67.3 \%$ knew about their modes of transmission. However, $49.8 \%$ considered the information to be insufficient.

Conclusions: This study revealed a low prevalence of parasitic infections in schoolchildren and favorable sanitation in their households. A high frequency of antiparasitic drug use without previous diagnosis by stool examination was reported.

KEY WORDS: parasites; intestinal parasites; child; student; basic sanitation; urban sanitation. 


\section{INTRODUÇÃO}

As condições de vida da população, como saneamento básico, higiene e nível socioeconômico são fatores determinantes para a transmissão de doenças, tais como as endoparasitoses ou parasitoses intestinais, que são consideradas um grande problema de saúde pública no mundo e uma das principais causas de morbidade e mortalidade humana. Estima-se que mundialmente mais de um bilhão de pessoas estejam infectadas por algum tipo de parasitose [1-5].

No Brasil há alta prevalência dessas afecções, e um grupo de risco são as crianças em idade escolar [6,7]. As condições de vida precárias a que muitas crianças estão sujeitas, a falta de hábitos corretos de higiene pessoal, o frequente contato com locais possivelmente contaminados, como solo, água e areia [8], além de um sistema imunológico imaturo [6,9], favorecem o desenvolvimento de parasitoses.

Um levantamento multicêntrico realizado no Brasil sobre parasitoses intestinais encontrou uma prevalência de $28,5 \%$ em escolares com idade de sete a 14 anos [10]. Estudos em diferentes locais do país mostram que há grande diferença na prevalência de parasitoses em escolares conforme a zona de moradia. Por exemplo, no sertão da Bahia foram observadas $70,7 \%$ de amostras positivas, enquanto um estudo na região nordeste obteve $36,5 \%$ de infestação por parasitas [3,11-13].

O objetivo deste estudo foi determinar a prevalência de parasitas intestinais em escolares do ensino fundamental de escolas municipais da zona urbana de Caxias do Sul, Rio Grande do Sul, Brasil, relacionando-a com as condições sanitárias nas quais se localizavam suas moradias e o conhecimento que os responsáveis relatavam ter sobre parasitoses.

\section{MÉTODOS}

Foi realizado um estudo transversal que teve como público alvo crianças do ensino fundamental da rede municipal de Caxias do Sul, Rio Grande do Sul, Brasil. O estudo foi aprovado pelo Comitê de Ética em Pesquisa da Faculdade da Serra Gaúcha sob o parecer 479.964.

Foram selecionadas 12 escolas localizadas em diferentes pontos da cidade, tanto em zonas carentes da periferia quanto zonas centrais, não sendo incluída a zona rural do município. O número amostral foi determinado por conveniência. Todos os estudantes que estavam em sala de aula no dia e turno previamente agendados com a direção da escola para realização da visita dos pesquisadores, foram convidados a participar da pesquisa. Foi ministrada uma pequena palestra sobre parasitoses com intuito de informá-los sobre a importância do assunto e sobre as formas de prevenção. Aos que aceitaram participar foi entregue um questionário, recipientes especiais para o exame parasitológico de fezes, juntamente com instruções para a coleta, e o Termo de Consentimento Livre e Esclarecido, os quais foram levados para sua residência. Foi solicitado que um dos pais ou responsáveis respondesse ao questionário e assinasse o termo de consentimento. Nos casos em que havia estudantes de uma mesma residência, o responsável respondeu a dois questionários, ou seja, um por criança. Foram realizadas duas visitas dos pesquisadores às escolas, uma para convidar os alunos a participarem da pesquisa e outra, normalmente dois dias após, para buscar as amostras e os questionários.

O questionário solicitava algumas informações sociodemográficas do familiar respondente e as seguintes questões: - Você sabe o que são parasitoses? - Você conhece os seus meios de transmissão? - Qual o meio pelo qual você obtém informações sobre o assunto? - As informações que você tem são suficientes? Foram solicitados, ainda, dados sobre história de parasitoses nos escolares, tratamentos realizados e exames aos quais já haviam sido submetidos. Também havia questões sobre saneamento básico, abastecimento de água, esgoto sanitário e coleta de lixo, com base no estudo de Andreazzi et al. [14], além de questões sobre controle de pragas (insetos e roedores) e captação de água das chuvas. Esse questionário completo foi validado por Cavagnolli et al. [15].

Após os pesquisadores recolheram as amostras de fezes nas escolas, as mesmas foram mantidas refrigeradas até sua análise dentro de 24 horas. Não foi utilizado nenhum tipo de conservante no frasco de coleta. As análises foram realizadas no Laboratório de Parasitologia do Instituto Integrado de Saúde da Faculdade da Serra Gaúcha, pela técnica de sedimentação espontânea. As amostras ficaram sedimentando por um prazo mínimo de duas horas. A técnica foi escolhida por ser de fácil execução e baixo custo e por ser indicada para a pesquisa de cistos, ovos e larvas. Além disso, pelo fato de poder ser utilizada uma grande quantidade de material fecal no processo, em contraste com a pequena quantidade utilizada em outras técnicas, essa técnica favorece a sensibilidade, mesmo quando o número de estruturas parasitárias é pequeno [16]. O material foi avaliado de acordo com suas características morfológicas e através da análise 
microscópica do sedimento, com lugol entre lâmina e lamínula, por dois observadores. Os resultados foram expressos informando o nome científico do parasita identificado ou comunicando que não foram observadas estruturas parasitárias na amostra analisada.

Os dados numéricos contínuos foram demonstrados em média e desvio padrão e os dados categóricos em frequências absolutas e percentuais. Para a comparação entre desfechos foi aplicado o teste de qui-quadrado ou exato de Fisher, considerando-se significativo $p \leq 0,05$. As análises foram realizadas com o programa IBM SPSS Statistics 20.0.

\section{RESULTADOS}

Das 12 escolas convidadas a participar da pesquisa, nove aceitaram participar, sendo oito localizadas em zona periférica da cidade e uma na área central. De 291 crianças cujos familiares foram entrevistados, 34 foram excluídas por não terem entregue a amostra de fezes, o questionário ou o termo de consentimento assinado. Portanto, foram estudados 257 escolares, entre os quais 149 (58\%) eram do sexo feminino. A idade média das crianças foi de $8,7 \pm 1,3$ anos, variando de cinco a 13 anos. Observou-se que a idade média dos responsáveis respondentes foi de $36,7 \pm 8,0$ anos, sendo a maioria do sexo feminino $(n=205 ; 79,8 \%)$. Dados sobre escolaridade dos responsáveis e conhecimento destes sobre os parasitas, assim como história prévia de parasitoses e tratamentos, estão descritos na Tabela 1.

Quanto às condições de saneamento básico em que a população estudada estava inserida, observouse que na grande maioria eram boas, no que se refere às variáveis pesquisadas (limpeza pública das ruas, captação de água das chuvas, coleta de lixo, esgoto e disponibilidade de água potável). A presença de insetos e roedores nas moradias foi relatada por $44,7 \%$ dos entrevistados. Não foram encontradas associações significativas entre as variáveis de saneamento e a presença de parasitoses intestinais nas crianças (Tabela 2).

Das amostras de fezes coletadas obteve-se resultado positivo em $15(5,9 \%)$ estudantes, todos esses infectados por protozoários. Desse total, foram observados cistos de Endolimax nana em $60 \%$ dos estudantes, de Entamoeba coli em 26,7\% e de Giardia lamblia em 13,3\%. Não houve associação significativa entre sexo, idade, escolaridade dos responsáveis ou uso de tratamento antiparasitário com o encontro de parasitas intestinais nos escolares.
Avaliando-se os questionários respondidos pelos responsáveis dos 15 escolares parasitados, observouse que todos habitavam em locais com limpeza de ruas e coleta de lixo; 14 desses relataram ter acesso à água potável; 13 moravam em área com tratamento de esgoto; oito responderam que havia captação da água das chuvas e cinco relataram existir pragas nas proximidades das suas residências.

Tabela 1. Escolaridade, relato de conhecimento sobre parasitoses pelos responsáveis, diagnóstico prévio e tratamento de parasitoses em escolares, obtidos por meio da aplicação de questionário. Caxias do Sul, Rio Grande do Sul, Brasil, 2014.

\begin{tabular}{|c|c|c|c|}
\hline Variável & Categoria & $\mathbf{n}$ & $(\%)$ \\
\hline \multirow{7}{*}{$\begin{array}{l}\text { Escolaridade do responsável } \\
\text { respondente }\end{array}$} & Fundamental incompleto & 63 & 24,5 \\
\hline & Fundamental completo & 30 & 11,7 \\
\hline & Médio incompleto & 22 & 8,6 \\
\hline & Médio completo & 73 & 28,4 \\
\hline & Superior incompleto & 16 & 6,2 \\
\hline & Superior completo & 20 & 7,8 \\
\hline & Não informado & 33 & 12,8 \\
\hline \multirow{2}{*}{$\begin{array}{l}\text { Sabem o que são } \\
\text { parasitoses? }\end{array}$} & Sim & 192 & 74,7 \\
\hline & Não & 65 & 25,3 \\
\hline \multirow{2}{*}{$\begin{array}{l}\text { Sabem os meios de } \\
\text { transmissão de parasitas? }\end{array}$} & Sim & 173 & 67,3 \\
\hline & Não & 84 & 32,7 \\
\hline \multirow{7}{*}{$\begin{array}{l}\text { Onde obtêm informações } \\
\text { sobre parasitas? }\end{array}$} & Profissionais da saúde & 111 & 43,2 \\
\hline & Televisão & 76 & 29,6 \\
\hline & Internet & 35 & 13,6 \\
\hline & Rádio & 5 & 1,9 \\
\hline & Jornal & 3 & 1,2 \\
\hline & Outros & 18 & 7 \\
\hline & Não informado & 9 & 3,5 \\
\hline \multirow{2}{*}{$\begin{array}{l}\text { São suficientes as } \\
\text { informações disponíveis } \\
\text { sobre parasitas? }\end{array}$} & Sim & 129 & 50,2 \\
\hline & Não & 128 & 49,8 \\
\hline \multirow{3}{*}{$\begin{array}{l}\text { A criança já recebeu } \\
\text { tratamento para parasitoses? }\end{array}$} & Sim & 217 & 84,4 \\
\hline & Não & 39 & 15,2 \\
\hline & Não sabe & 1 & 0,4 \\
\hline \multirow{5}{*}{$\begin{array}{l}\text { Frequência com que } \\
\text { a criança faz uso de } \\
\text { antiparasitários }\end{array}$} & Anualmente & 93 & 36,2 \\
\hline & Semestramente & 69 & 26,8 \\
\hline & Quando necessário & 42 & 16,3 \\
\hline & Biênio & 1 & 0,4 \\
\hline & Não sabe & 52 & 20,2 \\
\hline \multirow{4}{*}{$\begin{array}{l}\text { Princípio ativo de } \\
\text { medicamentos citados para } \\
\text { o tratamento de parasitoses }\end{array}$} & Albendazol & 27 & 10,5 \\
\hline & Mebendazol & 20 & 7,8 \\
\hline & Nitazoxanida & 17 & 6,6 \\
\hline & Não sabe & 193 & 75,1 \\
\hline
\end{tabular}


Tabela 2. Condições de saneamento básico das moradias de uma amostra de 257 escolares de Caxias do Sul, Rio Grande do Sul, Brasil e relação com a prevalência de parasitoses.

\begin{tabular}{|c|c|c|c|c|}
\hline $\begin{array}{c}\text { Variáveis de } \\
\text { saneamento ambiental }\end{array}$ & $\begin{array}{c}\text { Domicílios com presença } \\
\text { da variável } \\
\text { n (\%)* }\end{array}$ & $\begin{array}{c}\text { Crianças com exame } \\
\text { de fezes positivo } \\
\text { (n) }\end{array}$ & $\begin{array}{l}\text { Porcentagem de casos positivos } \\
\text { em relação a cada categoria da } \\
\text { variável de saneamento } \\
(\%)^{* *}\end{array}$ & p \\
\hline $\begin{array}{l}\text { Limpeza pública das ruas } \\
\text { Sim } \\
\text { Não }\end{array}$ & $\begin{array}{l}230(89,5) \\
27(10,5)\end{array}$ & $\begin{array}{l}15 \\
-\end{array}$ & $\begin{array}{c}6,5 \\
0\end{array}$ & $0,380+$ \\
\hline $\begin{array}{l}\text { Captação de água das chuvas } \\
\text { Sim } \\
\text { Não }\end{array}$ & $\begin{array}{l}180(70,0) \\
77(30,0)\end{array}$ & $\begin{array}{l}8 \\
7\end{array}$ & $\begin{array}{l}4,4 \\
9,0\end{array}$ & $0,538 \neq$ \\
\hline $\begin{array}{l}\text { Coleta pública de lixo } \\
\text { Sim } \\
\text { Não }\end{array}$ & $\begin{array}{l}255(99,2) \\
2(0,8)\end{array}$ & $\begin{array}{l}15 \\
--\end{array}$ & $\begin{array}{c}5,8 \\
0\end{array}$ & $0,886+$ \\
\hline $\begin{array}{l}\text { Coleta e tratamento de esgoto } \\
\text { Sim } \\
\text { Não }\end{array}$ & $\begin{array}{c}246(95,7) \\
11(4,3)\end{array}$ & $\begin{array}{c}13 \\
2\end{array}$ & $\begin{array}{c}5,2 \\
18,1\end{array}$ & $0,193+$ \\
\hline $\begin{array}{l}\text { Disponibilidade de água potável } \\
\text { Sim } \\
\text { Não }\end{array}$ & $\begin{array}{l}255(99,2) \\
2(0,8)\end{array}$ & $\begin{array}{c}14 \\
1\end{array}$ & $\begin{array}{c}5,5 \\
50,0\end{array}$ & $0,114+$ \\
\hline $\begin{array}{l}\text { Pragas (insetos, roedores) } \\
\text { Não } \\
\text { Sim }\end{array}$ & $\begin{array}{l}142(55,3) \\
115(44,7)\end{array}$ & $\begin{array}{c}10 \\
5\end{array}$ & $\begin{array}{l}7,0 \\
4,3\end{array}$ & $0,261 \neq$ \\
\hline
\end{tabular}

* Porcentagens calculadas sobre o total (257).

** Porcentagens calculadas sobre cada categoria das variáveis de saneamento.

+ Teste exato de Fisher.

₹ Teste do qui-quadrado.

\section{DISCUSSÃO}

No presente estudo, o percentual de amostras positivas difere dos resultados encontrados em outra pesquisa realizada em Caxias do Sul, que encontrou $58 \%$ de amostras positivas em escolares de 6 a 14 anos. Aquela pesquisa ocorreu nos anos de 1969 a 2004 e os autores mostraram que houve uma queda de prevalência de parasitoses no decorrer dos anos estudados [7]. Este fato está de acordo com o percentual encontrado no presente estudo, realizado em torno de 10 anos depois.

Outros estudos realizados em cidades das regiões Sul e Sudeste do Brasil mostraram prevalência maior de parasitoses, como ocorreu em Porto Alegre, RS, com 39\% [17] e em São Joaquim, SC, com 22,36\% de positividade em escolares [18]. Outros estudos nas mesmas regiões, entretanto, detectaram percentuais próximos ao do presente estudo, como em Osório, RS, com 10,3\% [19], em Estiva Gerbi, SP, com 11,5\% [20], em Sananduva, RS, com 8,1\% [21], em São Miguel do Oeste, SC, com 7,4\% [22] e em Concórdia, SC, com $12,6 \%$ [23] de amostras positivas.

Nota-se grande variação de prevalência de parasitoses em diferentes regiões do Brasil e mesmo dentro do Rio Grande do Sul, que podem ser explicadas por condições de saneamento básico e socioeconômicas das diferentes populações. Higiene inadequada, más condições de abastecimento de água, entre outros, são determinantes para a transmissão desses parasitas [24]. Em um estudo realizado em Coari, AM, foram observadas $73 \%$ de amostras positivas [25]. Naquela zona, apenas $6,5 \%$ da população estudada possui água encanada, 92\% utiliza água não tratada e $87,5 \%$ vive em meio a condições inadequadas de saneamento [26]. Estudo realizado no Ceará com 383 crianças identificou 233 parasitadas, sendo que 38\% das moradias dispunham apenas de água não tratada e somente $45 \%$ possuíam fossa séptica como forma de saneamento [6]. As boas condições sanitárias encontradas no presente estudo podem justificar a baixa prevalência de parasitoses nesta amostra.

Não foram encontradas associações entre condições de saneamento e presença de parasitoses nas crianças, possivelmente pelo tamanho amostral insuficiente, além do fato de que a grande maioria das moradias possuía ótimas condições de saneamento. Não foram pesquisadas, entretanto, as condições de saneamento das próprias escolas, onde os escolares passam grande parte do seu tempo.

Outro fator que pode influenciar a prevalência de parasitoses é o nível de educação da população. Uma pesquisa realizada em Manaus apresentou prevalência de $44,2 \%$ de parasitoses, sendo que $56,8 \%$ dos participantes possuíam ensino fundamental 
incompleto [27]. Em contraste, observou-se no presente estudo que apenas um quarto dos responsáveis não possuíam ensino fundamental completo. $\mathrm{Na}$ amostra entrevistada, a metade das pessoas referiam carecer de informações suficientes sobre parasitoses, apesar de em torno de $70 \%$ alegarem saber o que são parasitoses e terem conhecimento dos seus meios de transmissão. Limitações deste estudo, no que tange à avaliação sobre os conhecimentos dos responsáveis, incluíram $\mathrm{o}$ fato de o questionário ter sido preenchido em casa $\mathrm{e}$ de não conter perguntas que avaliassem objetivamente os conhecimentos dos respondentes, sendo esse dado autorrelatado.

Um ponto importante deste estudo é o fato de que $84,4 \%$ das crianças já haviam feito algum tratamento com vermífugo, no entanto, $71,6 \%$ nunca haviam realizado exame parasitológico de fezes. Esses dados indicam que não há orientações adequadas sobre o diagnóstico e o tratamento dessas doenças na população caxiense. $\mathrm{O}$ mesmo comportamento foi relatado em um estudo realizado em Assis, SP, onde os autores detectaram o hábito de fornecer antiparasitários como medida profilática [28]. É necessário um maior investimento em saúde pública, com programas de conscientização e educação sobre a automedicação para parasitoses, tanto para os responsáveis como para os escolares [29]. A falta de conhecimento no assunto pode trazer prejuízos à saúde do indivíduo, relacionados ao uso indevido de vermífugos.

Estudos relatam que uma das limitações encontradas em pesquisas envolvendo crianças é o baixo retorno nas coletas das amostras de fezes ou dados incompletos, que pode ser causado pelo constrangimento dos participantes ou a necessidade da ajuda dos pais para a coleta e o preenchimento do questionário $[19,30]$. Neste estudo apenas $22 \%$ dos estudantes foram excluídos por esses motivos. Quanto à prevalência das espécies encontradas (Endolimax nana $60 \%$, Entamoeba coli $26,7 \%$ e Giardia lamblia $13,3 \%$ ), a mesma é compatível com resultados obtidos em outros estudos das regiões Sul e Sudeste [21,31]. Além disso, também é semelhante aos resultados encontrados em escolares na região Nordeste, onde protozoários comensais foram os mais prevalentes (Entamoeba coli 35,8\% e Endolimax nana 13,6\%) [13]. A distribuição das parasitoses em relação ao sexo corrobora com dados obtidos em um estudo realizado em Porto Alegre, RS, o qual também não encontrou diferença significativa [17].

Pode-se concluir que a prevalência de parasitoses encontrada na amostra de escolares foi das menores em relação a outros estudos, principalmente os realizados em outras regiões do Brasil, embora ainda possa ser considerada uma prevalência relevante. Houve relato de grande frequência de uso de medicamentos antiparasitários sem diagnóstico prévio por exame de fezes. Foram identificados bons níveis de saneamento básico nas moradias desta população de Caxias do Sul, quando comparados aos de outras cidades, entretanto são necessários mais estudos sobre as condições sanitárias e de higiene das próprias escolas, as quais as crianças frequentam por longo período do dia.

\section{NOTA}

Declaração de conflitos de interesse

Os autores declaram não haver conflitos de iteresse relevantes ao conteúdo deste estudo.

\section{REFERÊNCIAS}

1. Tavares-Dias M, Grandini AA. Prevalência e aspectos epidemiológicos de enteroparasitoses na população de São José da Bela Vista, São Paulo. Rev Soc Bras Med Trop. 1999;32(1):63-5. http://dx.doi.org/10.1590/S0037-86821999000100012

2. Souza PAC, de Faro CCP, Pinheiro MS, de Rezende Neto JM, de Brito AMG. Ocorrência de enteroparasitoses em portadores de transtornos mentais assistidos na Clínica de Repouso São Marcello em Aracaju (SE). Rev Cien Saúde Col. 2010;15(supl 1):1081-4. http://dx.doi. org/10.1590/S1413-81232010000700015

3. Fonseca EOL, Teixeira MG, Barreto ML, Carmo EH, Costa MCN. Prevalência e fatores associados às geo-helmintíases em crianças residentes em municípios com baixo IDH no Norte e Nordeste brasileiros. Cad Saúde Publica. 2010;26(1):143-52. http://dx.doi.org/10.1590/ S0102-311X2010000100015

4. Basualdo JA, Córdoba MA, Luca MM, Ciarmela ML, Pezzani BC, Grenovero MS, Minvielle MC. Intestinal parasitoses and environmental factors in a rural population of Argentina, 2002-2003. Rev Inst de Med Trop S Paulo. 2007;49(4):251-5. http://dx.doi.org/10.1590/S003646652007000400011

5. Andrade E, Leite ICG, Rodrigues VdO, Cesca MG. Parasitoses intestinais: uma revisão sobre seus aspectos sociais, epidemiológicos, clínicos e terapêuticos. Rev APS. 2010;13(2):231-40.

6. Vasconcelos IAB, Oliveira JW, Cabral FRF, Coutinho HDM, Menezes IRA. Prevalência de parasitoses intestinais entre crianças de 4-12 anos no Crato, Estado do Ceará: um problema recorrente de saúde pública. Acta Sci Health Sci. 2011;33(1):35-41. 
7. Basso RMC, Silva-Ribeiro RT, Soligo DS, Ribacki SI, Callegari-Jacques SM, Zoppas B. Evolução da prevalência de parasitoses intestinais em escolares em Caxias do Sul, RS. Rev Soc Bras Med Trop. 2008;41(3):263-8. http://dx.doi.org/10.1590/S0037-86822008000300008

8. Araujo Filho HB, Carmo-Rodrigues MS, Mello CS, Melli LCF, Tahan S, Morais MBd. Parasitoses intestinais se associam a menores índices de peso e estatura em escolares de baixo estrato socioeconômico. Rev Paul Pediatr. 2011;29(4):521-8. http://dx.doi.org/10.1590/ S0103-05822011000400009

9. Oliveira Figueiredo MI, Wendt EW, Santos HT, Moreira CM. Levantamento sazonal de parasitos em caixas de areia nas escolas municipais de educação infantil em Uruguaiana, RS, Brasil. Rev Patol Trop. 2012;41(1):36-46. http://dx.doi.org/10.5216/rpt.v41i1.17744

10. Mamus CNC, Moitinho ACC, Grube CC, Melo EM, Weiler EB, Abreu CA, Beltrão L, Soares PB, Beltrame S, Ribeiro S, Aleixo DL. Enteroparasitoses em um centro de educação infantil do município de Iretama/PR. SaBios. 2008;3(2):39-44.

11. Santos-Júnior GO, Silva MM, Santos FLN. Prevalência de enteroparasitoses em crianças do sertão baiano pelo método de sedimentação espontânea. Rev Patol Trop. 2006;35(3):233-40.

12. Prado MdS, Barreto ML, Strina A, Faria JAS, Nobre AA, Jesus SR. Prevalência e intensidade da infecção por parasitas intestinais em crianças na idade escolar na Cidade de Salvador (Bahia, Brasil). Rev Soc Bras Med Trop. 2001;34(1):99-101.

13. Alves JR, Macedo HW, Ramos Jr AN, Ferreira LF, Gonçalves MLC, Araújo A. Parasitoses intestinais em região semi-árida do Nordeste do Brasil: resultados preliminares distintos das prevalências esperadas. Cad Saúde Pública. 2003;19(2):667-70. http://dx.doi.org/10.1590/ S0102-311X2003000200034

14. Andreazzi MAR, Barcellos C, Hacon S. Velhos indicadores para novos problemas: a relação entre saneamento e saúde. Rev Panam Salud Publ. 2007;22(3):211-7. http://dx.doi.org/10.1590/S1020-49892007000800008

15. Cavagnolli NI, Camello JT, Tesser S, Poeta J, Rodrigues AD. Prevalência de enteroparasitoses e análise socioeconômicas de escolares em Flores da Cunha-RS. Rev Patol Trop. 2015;44(3):312-22. http://dx.doi.org/10.5216/rpt.v44i3.38018

16. De Carli, GA. Parasitologia Clínica. 2aed. São Paulo: Atheneu; 2008.

17. Roque FC, Borges FK, Signori LGH, Chazan M, Pigatto T, Coser TA, Mezzari A, Wiebbelling AMP. Parasitos intestinais: prevalência em escolas da periferia de Porto Alegre-RS. Newslab. 2005;69:152-62.

18. Schmitt ML, Paes MAS. Prevalência de parasitoses intestinais em escolares no município de São Joaquim, SC. Rev Bras Anal Clin. 1997;29(4):215-6.

19. Abrahão ST. Prevalência de enteroparasitoses em escolares no município de Osório, RS. NewsLab. 2013;119:148-54.

20. Ferreira GR, Andrade CFS. Alguns aspectos socioeconômicos relacionados a parasitoses intestinais e avaliação de uma intervenção educativa em escolares de Estiva Gerbi, SP. Rev Soc Bras Med Trop. 2005;38(5):402-5. http://dx.doi.org/10.1590/S0037-86822005000500008

21. Bellin M, Grazziotin NA. Prevalência de Parasitos Intestinais no município de Sananduva/RS. News Lab. 2011;104:116-22.

22. Seger J, Souza WM, Marangoni JCF, Maschio VJ, Chielli EO. Prevalência de parasitas intestinais na população do Bairro Salete, município de São Miguel do Oeste, SC.

23. Tietz SM, Bandeira C, Marinho RQ. Prevalência de enteroparasitoses em Concórdia, Santa Catarina, Brasil. Parasit Latinoam. 2005; 60(1-2):78-81.

24. Ludwig KM, Frei F, Alvares Filho F, Ribeiro-Paes JT. Correlação entre condições de saneamento básico e parasitoses intestinais na população de Assis, Estado de São Paulo. Rev Soc Bras Med Trop. 1999;32(5):547-55. http://dx.doi.org/10.1590/S0037-86821999000500013

25. Silva EF, Silva EB, Almeida KdS, Sousa JJN, Freitas FLdC. Enteroparasitoses em crianças de áreas rurais do munícipio de Coari, Amazonas, Brasil. Rev Patol Trop. 2009;38(1):35-43. http://dx.doi.org/10.5216/rpt.v38i1.6219

26. Santos Fd, Gama A, Fernandes A, Reis-Jr J, Guimarães J. Prevalência de enteroparasitismo em crianças de comunidades ribeirinhas do município de Coari, no médio Solimões, Amazonas, Brasil. Rev Pan-Amaz Saude. 2010;1(4):23-8. http://dx.doi.org/10.5123/S217662232010000400004

27. Visser S, Giatti LL, Carvalho RACd, Guerreiro JCH. Estudo da associação entre fatores socioambientais e prevalência de parasitose intestinal em área periférica da cidade de Manaus (AM, Brasil). Cien Saúde Colet. 2011;16(8):3481-92. http://dx.doi.org/10.1590/S141381232011000900016

28. Frei F, Juncansen C, Ribeiro-Paes JT. Levantamento epidemiológico das parasitoses intestinais: viés analítico decorrente do tratamento profilático. Cad Saúde Pública. 2008;24(12):2919-25. http://dx.doi.org/10.1590/S0102-311X2008001200021

29. Silva JC, Furtado LFV, Ferro TC, Carvalho Bezerra K, Borges EP, Melo ACFL. Parasitismo por Ascaris lumbricoidese e seus aspectos epidemiológicos em crianças do estado do Maranhão. Rev Soc Bras Med Trop. 2011;44(1):100-2. http://dx.doi.org/10.1590/S003786822011000100022

30. Castro AZ, Viana JD, Penedo AA, Donatele DM. Levantamento das parasitoses intestinais em escolares da rede pública na cidade de Cachoeiro de Itapemirim-ES. News Lab. 2004;64:140-4.

31. Biasi LA, Tacca JA, Navarini M, Belusso R, Nardino A, Santolin JC, Bernardon V, Jaskulski MR. Prevalência de enteroparasitoses em crianças de entidade assistencial de Erechim/RS. Perspect [Internet]. 2010 [cited 2016]:173-9. Available from: http://www.uricer.edu.br/ site/pdfs/perspectiva/125_85.pdf $C$ 